\title{
CLUSTER BASED ROUTING MECHANISM FOR OPTIMIZED DATA DISTRIBUTION IN MOBILE COMPUTING
}

\author{
Faizul Navi Khan ${ }^{1}$, Kapil Govil ${ }^{2}$, Alok Agarwal ${ }^{3}$ \\ ${ }^{I}$ Teerthanker Mahaveer University, Moradabad, UP, INDIA \\ ${ }^{2}$ Teerthanker Mahaveer University, Moradabad, UP, INDIA \\ ${ }^{3}$ J.P. Institute of Eng. and Tech., Meerut UP, INDIA
}

\begin{abstract}
Data distribution in mobile computing network is an increasingly attractive mechanism for communication with remote, non-fixed or mobile devices. Wireless technology enhancement makes possible to collect and distribute data virtually anywhere regardless location of the user in efficient and reliable manner. However, the frequency of transmission and amount of data sent in each exchange can have significant time, cost, and reliability and performance impact on Mobile computing network. In mobile computing network users can keep the information handy and also dynamic access to the application by using mobile devices i.e. Smart phones, palmtop, laptop and tablets. An application distributes data on various processing units in mobile computing network to process by using a routing mechanism. An optimize data distribution is required among the mobile computing network devices in order to enhance the performance. To achieve optimal data distribution an efficient a routing technique proposes here through this research paper. The performance of a mobile computing network is depends on employed routing technique by the network and it also plays a significant role to improve the performance in Mobile computing network. In mobile computing network multiple data route exists for data transmission and data distributes on processing units through the most feasible route in order to achieve minimum transmission cost, time and maximum reliability. This research paper demonstrates a routing technique with the help of clusters to distribute data packets among the devices within the mobile computing network.
\end{abstract}

Keywords - Data distribution, Mobile Computing, Optimization, Performance, Routing

$* * *$

\section{INTRODUCTION}

In mobile computing network devices are self-organized and use wireless communication medium for interaction between them. Data distribution is one of the main and critical functions of Mobile computing network and it comprises two phases, the route finding and the data transmission. Routing solutions should be compatible with the nature of the network, and aim at minimizing control traffic, to preserve both bandwidth and energy at devices. Data distribution during the execution of any application among the processing units in form of packets is a basic and primary activity in mobile computing. This activity is performed using a routing scheme, which is a mechanism working in a distributed fashion for routing data packets in Mobile computing.

It is very useful for the business organization, government sectors and educational institutions. In past network was use fixed wired connections that was having limitations in regards of locations. In wired network user should reach the location where network connected terminal is present, then only user was enabled to access network resources. Mobile computing removes all such kind of constraints and provides hassle free network application access anytime and anywhere due to the tremendous improvement in wireless technologies, it provides the facility continuous network connectivity to users regardless of their location. Exchange of information and data is also being the part of mobile computing between processors in form of data packets. This activity is performed by using a routing technique, basically it is a communication mechanism working in a distributed manner for data routing in Mobile Computing.

Due to the many physical and environmental constrains network routing is become a challenging task for the researchers. It also helpful to improves the performance of Mobile Computing network. Finding an optimal routing solution becomes more critical when the more than one transmission paths are available for a single data packets transmission in a mobile computing network. In such scenario extra effort would be needed towards finding an effective routing solution to select most appropriate transmission route to transmit data packets in mobile computing network for performance enhancement. This research paper present a design of routing technique that would distribute the data by selecting the most suited path in a Mobile computing network where the numbers of data packets ' $\mathrm{m}$ ' will distribute on numbers of processors ' $\mathrm{n}$ ' (where $\mathrm{m}>\mathrm{n}$ ). Routing technique present in this research paper find the optimal route for distribution of data packets, routing technique will satisfy time, cost and reliability constraint here, it means performance will measure in terms of transmission time, cost and reliability or i.e. minimum transmission time, cost and maximum reliability of data packets.

This research paper considers a problem of data distribution in Mobile computing network, where the multiple mobile 
devices are using application running in same network domain. In a mobile computing network a source device send across data packets using application and these data packets distributes over the processing units available in network to get process using routing algorithm. For optimal data distribution process especially where multiple transmission route exist for single data packets, routing algorithm will find optimal path to minimize transmission time, cost or maximize reliability for a data packet in mobile computing. Some of the routing mechanism have been reported in the literature, such as Content distribution [1], Routing protocol [2, 5, 17], wireless sensor networks [3, 7], routing mechanism [4, 22], Trust based routing [6], Task scheduling technique [8], Task assignment model [9], Static approach [10, 13], routing strategy [11], Task allocation scheme [12], shortest multipath source routing scheme [14], Routing techniques $[15,19,24]$, communication reliability [16], energy efficient distributed protocol [18], job scheduling in mobile grids [20], data distribution [21] and Packet retransmission method [23]. This research paper proposes a routing solution by implementing a routing technique to get maximum optimization of data distribution for enhance the performance of Mobile Computing network.

\section{NOTATIONS}

d data packet

n Number of Processing units

$\mathrm{m}$ number of data packets

TCTR Transmission cost time and Reliability

CTCTR Cluster Transmission cost time and

Reliability

MTCTR Modified Transmission cost time and reliability.

\section{OBJECTIVE}

This research paper proposes routing solutions to discover an optimal route in order to achieve optimal data distribution in mobile computing network. The routing technique will be dealing with time, cost and reliability constraints of the network i.e. minimizing transmission cost time or maximizing reliability. To speed up data distribution an effective routing technique is always require and it will also help to improve the performance of mobile computing network. In network architecture applications data transmits between server and clients applications. In a mobile computing network application data packets are require to distribute or transmit by using a transmission route and such routes are maintained in routing table. These routing tables contained all states regarding the transmission time cost and reliability among the various communication links present within a mobile computing network. By using such information available in routing tables, routing technique selects the most appropriate route in terms of minimum transmission time cost or maximum reliability of data packets. This research paper provides a solution to solve the problem of data distribution in mobile computing network by optimizing routing techniques in order to achieve optimal data distribution. The nature of routing technique in data transmission is static. Routing technique will also ensure the transmission of all data packets at destination in optimize manner. In this paper performance is measured in term of time cost and reliability i.e. minimum transmission time cost or maximum reliability.

\section{TECHNIQUE}

This research paper chosen a problem of data distribution in mobile computing network where a set $D=\left\{d_{1}, d_{2}\right.$, $\left.\mathrm{d}_{3}, \ldots \ldots \ldots \mathrm{d}_{\mathrm{m}}\right\}$ of ' $\mathrm{m}$ ' data packets required to transmit on a set $P=\left\{p_{1}, p_{2}, p_{3}, \ldots \ldots \ldots p_{n}\right\}$ of ' $n$ ' processing units. Each data packet has $n$ number of transmission route in which routing technique will choose most appropriate route for data distribution. Every data packet has also contains some number of sub data packets known as data signals. Transmission time, cost and reliability are known for each data signals to reach at processing units in mobile computing network and arrange in TCTR. Communications between the data signals is also consider here, it will be either 1 or 0 , where 1 represent communication between the data signals while 0 represent no communication. That is given in communication matrix namely $\mathrm{CM}$ (,) of order $\mathrm{m} \mathrm{x}$ $\mathrm{m}$. Clusters for the data signals would be formed for each data signals based on the communication and the number of data signals in cluster will be $[\mathrm{m} / \mathrm{n}]$ in case $\mathrm{m}$ is even otherwise number of data signals will be $[m+1 / n]$ in a cluster. The employed routing technique re-arrange the set of clusters and get $n$ number of ordered pair and none of the data signals will be repeated in a cluster. Minimum number of data packet in any cluster should be 1, remaining ordered pair may be skipped. After these steps n numbers of clusters are formed for $\mathrm{n}$ numbers of processing units. Data signal clusters will be arrange in Cluster Transmission Cost Time and Reliability Matrix (CTCTR), and routing technique will calculate average column wise for each column. Calculated average values are compare with CTCTR in vertical manner for each element and search for the minimum value for transmission cost, time or maximum value for data signal reliability for processing unit and route the data cluster to destination point. The process is repeated until all data cluster would be routed to distribute in Mobile computing network.

The function to calculate overall time [Etime], cost [Ecost] and reliability [Ereilability] is given here:

$$
\begin{aligned}
\text { Etime } & =\left[\sum_{i=1}^{n}\left\{\sum_{i=1}^{n} E_{i j} X_{i j}\right\}\right] \\
\text { Ecost } & =\left[\sum_{i=1}^{n}\left\{\sum_{i=1}^{n} E C_{i j} X_{i j}\right\}\right] \\
\text { Ereliablity } & =\left[\prod_{i=1}^{n}\left\{\sum_{i=0}^{n} E_{i j} X_{i j}\right\}\right]
\end{aligned}
$$




\section{ALGORITHM}

1. Start Algorithm

2. Read the number of data packets in $m$

3. Read the number of processing units in $n$

4. Store Data Signals and Transmission Time, cost and reliability into Matrix TCTR (,) in order of $\mathrm{m} x \mathrm{n}$.

5. Store Communication Matrix (,) in order of $\mathrm{m} x \mathrm{~m}$.

6. Based on Communication matrix formed data cluster having $\mathrm{m} / \mathrm{n}$ numbers of data signals

7. Clubbing the values of Data signals present in Data cluster and derived a new Cluster Transmission Cost, Time and Reliability Matrix (CTCTR) and also set routing status as false for each cluster.

8. Calculate average () for each Column in vertical manner

9. While (All data clusters! = routed)

\{

i. Find minimum value for cost, time or maximum for reliability in vertical manner for each processing node

ii. $\quad$ Check routed status

iii. If routed status $=$ false

iv. Route the eligible data signals to available transmission route and set route status as TRUE.

v. Else

vi. $\quad$ search for next appropriate value

\}

10. State the results

11. End of algorithm

\section{IMPLEMENTATION}

This research paper solve a problem of data distribution in mobile computing network which consist a set $\mathrm{P}$ of 3 processing units $\left\{\mathrm{p}_{1}, \mathrm{p}_{2}, \mathrm{p}_{3}\right\}$ with different processing capacity and a set $D$ of 3 data packets $\left\{d_{1}, d_{2}, d_{3}\right\}$. Each data packet has also contained some data signals. These signals are required to distribute on processing units using routing technique that will discovers appropriate transmission routes as mentioned in Figure 1.

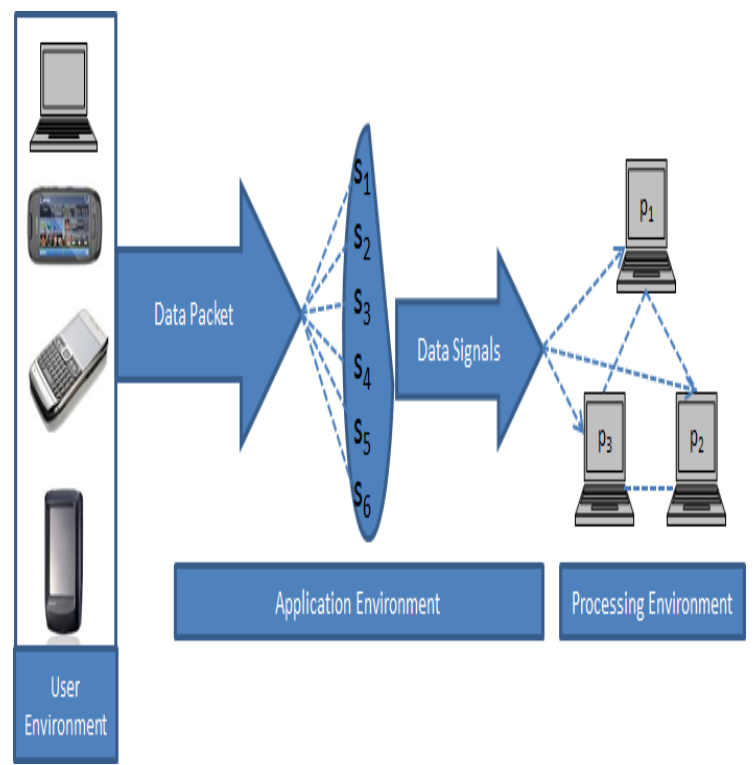

Fig 1: Data signals distribution in Mobile computing

Table 1: Data packets distributed in data signals

\begin{tabular}{|l|l|}
\hline $\mathrm{d}_{1}$ & $\left\{\mathrm{~s}_{11}, \mathrm{~s}_{12}, \mathrm{~s}_{13}, \mathrm{~s}_{14}, \mathrm{~s}_{15}\right\}$ \\
\hline $\mathrm{d}_{2}$ & $\left\{\mathrm{~s}_{21}, \mathrm{~s}_{22}, \mathrm{~s}_{23}, \mathrm{~s}_{24}, \mathrm{~s}_{25}, \mathrm{~s}_{26}\right\}$ \\
\hline $\mathrm{d}_{3}$ & $\left\{\mathrm{~s}_{31}, \mathrm{~s}_{32}, \mathrm{~s}_{33}, \mathrm{~s}_{34}, \mathrm{~s}_{35}, \mathrm{~s}_{36}, \mathrm{~s}_{37}, \mathrm{~s}_{38}\right\}$ \\
\hline
\end{tabular}

Each data packets contained different individual components which are known as signals. Transmission time $(\mathrm{t})$, transmission cost (c) and data packets reliability (r) of each data signals to each processing units are known and mentioned in Transmission Cost Time Reliability (TCTR) matrix as mentioned in Table 2

Table 2: Transmission Time Cost and Reliability Matrix

\begin{tabular}{|l|l|l|l|l|}
\hline \multirow{2}{*}{ Packets } & Processors & $\mathrm{p}_{1}$ & $\mathrm{p}_{2}$ & $\mathrm{p}_{3}$ \\
\hline $\mathrm{d}_{1}$ & Signals & $\mathrm{t}-\mathrm{c}-\mathrm{r}$ & $\mathrm{t}-\mathrm{c}-\mathrm{r}$ & $\mathrm{t}-\mathrm{c}-\mathrm{r}$ \\
\cline { 2 - 5 } & $\mathrm{s}_{11}$ & $10-105-0.949151$ & $30-125-0.959125$ & $09-130-0.979458$ \\
\cline { 2 - 5 } & $\mathrm{s}_{12}$ & $15-112-0.949429$ & $40-145-0.969157$ & $08-125-0.969425$ \\
\cline { 2 - 5 } & $\mathrm{s}_{13}$ & $08-118-0.997423$ & $15-115-0.979325$ & $15-170-0.929418$ \\
\cline { 2 - 5 } & $\mathrm{s}_{14}$ & $07-122-0.969218$ & $20-120-0.969414$ & $09-100-0.939458$ \\
\cline { 2 - 5 } & $\mathrm{s}_{15}$ & $20-108-0.969451$ & $08-140-0.979480$ & $10-115-0.992450$ \\
\hline $\mathrm{d}_{2}$ & $\mathrm{~S}_{21}$ & $15-100-0.999112$ & $19-220-0.971124$ & $17-140-0.991418$ \\
\cline { 2 - 5 } & $\mathrm{S}_{22}$ & $14-110-0.999149$ & $21-230-0.982152$ & $18-135-0.999429$ \\
\cline { 2 - 5 } & $\mathrm{S}_{23}$ & $20-120-0.999533$ & $15-200-0.979317$ & $15-170-0.999418$ \\
\cline { 2 - 5 } & $\mathrm{s}_{24}$ & $12-090-0.973218$ & $20-180-0.999419$ & $23-150-0.999413$ \\
\cline { 2 - 5 } & $\mathrm{s}_{25}$ & $22-140-0.929431$ & $32-220-0.989411$ & $26-165-0.999414$ \\
\cline { 2 - 5 } & $\mathrm{s}_{26}$ & $30-130-0.979328$ & $21-215-0.969019$ & $28-155-0.998226$ \\
\hline \multirow{3}{*}{$\mathrm{d}_{3}$} & $\mathrm{~s}_{31}$ & $11-105-0.999863$ & $22-112-0.999503$ & $20-155-0.998829$ \\
\cline { 2 - 5 } & $\mathrm{s}_{32}$ & $18-110-0.989733$ & $21-132-0.997136$ & $19-115-0.989449$ \\
\cline { 2 - 5 } & $\mathrm{s}_{33}$ & $25-130-0.999235$ & $15-120-0.997125$ & $18-115-0.989755$ \\
\hline
\end{tabular}




\begin{tabular}{|l|l|l|l|l|}
\hline \multirow{2}{*}{} & $\mathrm{s}_{34}$ & $21-120-0.999521$ & $25-130-0.999558$ & $20-105-0.997986$ \\
\cline { 2 - 5 } & $\mathrm{s}_{35}$ & $30-105-0.999631$ & $30-150-0.989537$ & $25-165-0.999358$ \\
\cline { 2 - 5 } & $\mathrm{s}_{36}$ & $35-125-0.999632$ & $20-120-0.997972$ & $28-110-0.999124$ \\
\cline { 2 - 5 } & $\mathrm{s}_{37}$ & $33-125-0.999478$ & $29-170-0.998903$ & $20-115-0.999754$ \\
\cline { 2 - 5 } & $\mathrm{s}_{38}$ & $10-155-0.999268$ & $22-152-0.998458$ & $23-125-0.996371$ \\
\hline
\end{tabular}

To represent all the three constraints i.e. transmission time, cost and reliability, TCTR matrix are considering that data packet $\mathrm{d}_{1}$ is based on transmission time ( $\mathrm{t}$ ) (it may be transmission cost or reliability), data packet $\mathrm{d}_{2}$ is based on transmission cost (c) (it may be transmission time and reliability) and data packet $\mathrm{d}_{3}$ is based on data signal reliability (r) (it may be transmission time and transmission cost). In result a new matrix named MTCTR can be derived by using TCTR, In MTCTR data packet $\mathrm{d}_{1}$ will represent transmission time $(\mathrm{t}), \mathrm{d}_{2}$ data packet transmission cost $(\mathrm{c})$ and $\mathrm{d}_{3}$ data packet reliability (r). New matrix MTCTR represent as Table 3:

Table 3: Modified Transmission Time Cost and Reliability Matrix

\begin{tabular}{|c|c|c|c|c|}
\hline & Processors & $\mathrm{p}_{1}$ & $\mathrm{p}_{2}$ & $\mathrm{p}_{3}$ \\
\hline Packets & Signals & $\mathrm{t}-\mathrm{c}-\mathrm{r}$ & $\mathrm{t}-\mathrm{c}-\mathrm{r}$ & $\mathrm{t}-\mathrm{c}-\mathrm{r}$ \\
\hline \multirow[t]{5}{*}{$\mathrm{d}_{1}$} & $\mathrm{~s}_{11}$ & $10-\ldots-\ldots$ & $30-\ldots-\ldots$ & $09-\ldots-\ldots$ \\
\hline & $\mathrm{S}_{12}$ & $15-\ldots-\ldots$ & $40-\ldots-\ldots$ & $08-\ldots-\ldots$ \\
\hline & $\mathrm{S}_{13}$ & $08-\ldots-\ldots$ & $15-\ldots-\ldots$ & $15-\ldots-\ldots$ \\
\hline & $\mathrm{s}_{14}$ & $07-\ldots-\ldots$ & $20-\ldots-\ldots$ & $09-\ldots-\ldots$ \\
\hline & $\mathrm{S}_{15}$ & $20-\ldots-\ldots$ & $08-\ldots-\ldots$ & $10-\ldots-\ldots$ \\
\hline \multirow[t]{6}{*}{$\mathrm{d}_{2}$} & $\mathrm{~S}_{21}$ & $\ldots-100-\ldots$ & $\ldots-220-\ldots$ & $\ldots-140-\ldots$ \\
\hline & $\mathrm{S}_{22}$ & $\ldots-110-\ldots$ & $\ldots-230-\ldots$ & $\ldots-135-\ldots$ \\
\hline & $\mathrm{S}_{23}$ & $\ldots-120-\ldots$ & $\ldots-200-\ldots$ & $\ldots-170-\ldots$ \\
\hline & $\mathrm{S}_{24}$ & $\ldots-090-\ldots$ & $\ldots-180-\ldots$ & $\ldots-150-\ldots$ \\
\hline & $\mathrm{S}_{25}$ & $\ldots-140-\ldots$ & $\ldots-220-\ldots$ & $\ldots-165-\ldots$ \\
\hline & $\mathrm{S}_{26}$ & $\ldots-130-\ldots$ & $\ldots-215-\ldots$ & $\ldots-155-\ldots$ \\
\hline \multirow[t]{8}{*}{$d_{3}$} & $\mathrm{~s}_{31}$ & $\ldots-\ldots-0.999863$ & $\ldots-\ldots-0.999503$ & $\ldots-\ldots-0.998829$ \\
\hline & $\mathrm{s}_{32}$ & $\ldots-\ldots-0.989733$ & $\ldots-\ldots-0.997136$ & ..-...-0.989449 \\
\hline & $\mathrm{s}_{33}$ & $\ldots-\ldots-0.999235$ & $\ldots-\ldots-0.997125$ & $\ldots-\ldots-0.989755$ \\
\hline & $\mathrm{s}_{34}$ & $\ldots-\ldots-0.999521$ & $\ldots-\ldots-0.999558$ & $\ldots-\ldots-0.997986$ \\
\hline & $\mathrm{s}_{35}$ & ...-...-0.999631 & $\ldots-\ldots-0.989537$ & ...-...-0.999358 \\
\hline & $\mathrm{S}_{36}$ & $\ldots-\ldots-0.999632$ & $\ldots-\ldots-0.997972$ & ...-..-0.999124 \\
\hline & $\mathrm{S}_{37}$ & $\ldots-\ldots-0.999478$ & $\ldots-\ldots-0.998903$ & $\ldots-\ldots-0.999754$ \\
\hline & $\mathrm{S}_{38}$ & $\ldots-\ldots-0.999268$ & $\ldots-\ldots-0.998458$ & $\ldots-\ldots-0.996371$ \\
\hline
\end{tabular}

MTCTR matrix can be represent into three different tables for each constraint i.e. Table 4 for transmission time, Table 5 for transmission cost and Table 6 for reliability in order to demonstrate all the three constraints of transmission i.e. time cost and reliability in Mobile Computing Network (MCN)
Table 4: Transmission Time

\begin{tabular}{|l|l|l|l|l|l|}
\hline & $\mathrm{s}_{11}$ & $\mathrm{~s}_{12}$ & $\mathrm{~s}_{13}$ & $\mathrm{~s}_{14}$ & $\mathrm{~s}_{15}$ \\
\hline $\mathrm{p}_{1}$ & 10 & 15 & 08 & 07 & 20 \\
\hline $\mathrm{p}_{2}$ & 30 & 40 & 15 & 20 & 08 \\
\hline $\mathrm{p}_{3}$ & 09 & 08 & 15 & 09 & 10 \\
\hline
\end{tabular}

Table 5: Transmission Cost

\begin{tabular}{|l|l|l|l|l|l|l|}
\hline & $\mathrm{s}_{21}$ & $\mathrm{~s}_{22}$ & $\mathrm{~s}_{23}$ & $\mathrm{~s}_{24}$ & $\mathrm{~s}_{25}$ & $\mathrm{~s}_{26}$ \\
\hline $\mathrm{p}_{1}$ & 100 & 110 & 120 & 090 & 140 & 130 \\
\hline $\mathrm{p}_{2}$ & 220 & 230 & 200 & 180 & 220 & 215 \\
\hline $\mathrm{p}_{3}$ & 140 & 135 & 170 & 150 & 165 & 155 \\
\hline
\end{tabular}


Table 6: Reliability

\begin{tabular}{|l|l|l|l|l|l|l|l|l|}
\hline & $\mathrm{s}_{31}$ & $\mathrm{~s}_{32}$ & $\mathrm{~s}_{33}$ & $\mathrm{~s}_{34}$ & $\mathrm{~s}_{35}$ & $\mathrm{~s}_{36}$ & $\mathrm{~s}_{37}$ & $\mathrm{~s}_{38}$ \\
\hline $\mathrm{p}_{1}$ & 0.999863 & 0.989733 & 0.999235 & 0.999521 & 0.999631 & 0.999632 & 0.999478 & 0.999268 \\
\hline $\mathrm{p}_{2}$ & 0.999503 & 0.997136 & 0.997125 & 0.999558 & 0.989537 & 0.997972 & 0.998903 & 0.998458 \\
\hline $\mathrm{p}_{3}$ & 0.998829 & 0.989449 & 0.989755 & 0.997986 & 0.999358 & 0.999124 & 0.999754 & 0.996371 \\
\hline
\end{tabular}

This research paper initially considers Table 4 for data distribution in $\mathrm{MCN}$ to represent a optimized routing mechanism. Table 4 represent 5 data signals belongs to data packets $d_{1}$ and these data signals need to distribute on 3 processing units. As per the present scenario, each data signal has 3 valid transmission routes to distribute and routing mechanism will choose any one of them, whichever is the most appropriate or optimize.

This research paper also considers communication between data signals and shown in Figure 2.

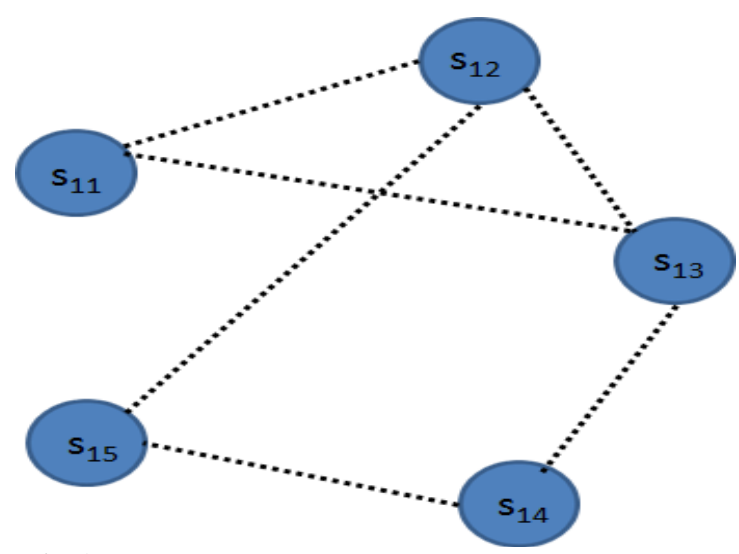

Fig 2: Communication graph between data signals

Communication table between data signals represents in Table 7 here.

Table 7: Communication table between Data Signals

\begin{tabular}{|l|l|l|l|l|l|l|}
\hline $\mathrm{CM}()=$, & & $\mathrm{s}_{11}$ & $\mathrm{~s}_{12}$ & $\mathrm{~s}_{13}$ & $\mathrm{~s}_{14}$ & $\mathrm{~s}_{15}$ \\
\cline { 2 - 7 } & $\mathrm{s}_{11}$ & 0 & 1 & 1 & 0 & 0 \\
\cline { 2 - 7 } & $\mathrm{s}_{12}$ & & 0 & 1 & 0 & 1 \\
\cline { 2 - 7 } & $\mathrm{s}_{13}$ & & & 0 & 1 & 0 \\
& & & & & & \\
\cline { 2 - 7 } & $\mathrm{s}_{14}$ & & & & 0 & 1 \\
\hline & $\mathrm{s}_{15}$ & & & & & 0 \\
\hline
\end{tabular}

By considering communication matrix CM () as mentioned in Table 7 the following data signal clusters will form:

For data signals $\mathrm{s}_{11}: \quad\left\{\left(\mathrm{s}_{11} * \mathrm{~s}_{12}\right),\left(\mathrm{s}_{11} * \mathrm{~s}_{13}\right)\right\}$

For data signals $s_{12}: \quad\left\{\left(s_{12} * s_{13}\right),\left(s_{12} * s_{15}\right)\right\}$

For data signals $\mathrm{s}_{13}: \quad\left\{\left(\mathrm{s}_{13} * \mathrm{~s}_{15}\right)\right\}$

For data signals $\mathrm{s}_{14}: \quad\left\{\left(\mathrm{s}_{14} * \mathrm{~s}_{15}\right)\right\}$

For data signals ${ }_{15}$ :

From the above sets of clusters by neglecting repeated data signal or ordered pair, three final distinct data cluster would be select $\left(\mathrm{s}_{1} * \mathrm{~s}_{3}\right),\left(\mathrm{s}_{2} * \mathrm{~s}_{5}\right) \&\left(\mathrm{~s}_{4}\right)$ :

Cluster $\mathrm{Cl}_{1}-\left\{\mathrm{s}_{11} * \mathrm{~s}_{13}\right\}$

Cluster $\mathrm{Cl}_{2}-\left\{\mathrm{s}_{12} * \mathrm{~s}_{15}\right\}$

Cluster $\mathrm{Cl}_{3}-\left\{\mathrm{s}_{14}\right\}$
This research paper will implement another matrix by using the data clusters instead of single data signal namely Cluster Transmission Time Matrix CTTM [,] from the Table 4, these data clusters will also contain their routing status, initially set to 0 as mentioned in Table 8 .

Table 8: Cluster Transmission Time Matrix

\begin{tabular}{|c|c|c|c|c|}
\hline \multirow[t]{4}{*}{ CTTM ()$=$} & Clusters & $\mathrm{p}_{1}$ & $\mathrm{p}_{2}$ & $\mathrm{p}_{3}$ \\
\hline & $\underset{\text { routing }=0}{\mathrm{Cl}_{1}\left\{\mathrm{~s}_{11} * \mathrm{~s}_{13}\right\}}$ & 18 & 45 & 24 \\
\hline & 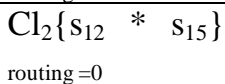 & 35 & 48 & 18 \\
\hline & $\mathrm{Cl}_{3}\left\{\mathrm{~s}_{14}\right\}_{\text {routing }=0}$ & 07 & 20 & 09 \\
\hline
\end{tabular}

As per the employed routing mechanism here, average will be calculated for each column as mentioned in Table 9:

Table 9: Average for each column

\begin{tabular}{|c|c|c|c|c|}
\hline \multirow[t]{5}{*}{ CTTM ()$=$} & Clusters & $\mathrm{p}_{1}$ & $\mathrm{p}_{2}$ & $\mathrm{p}_{3}$ \\
\hline & $\begin{array}{l}\mathrm{Cl}_{1}\left\{\mathrm{~s}_{11} * \mathrm{~s}_{13}\right\} \\
\text { routing }=0\end{array}$ & 18 & 45 & 24 \\
\hline & 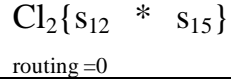 & 35 & 48 & 18 \\
\hline & $\begin{array}{l}\mathrm{Cl}_{3}\left\{\mathrm{~s}_{14}\right\} \\
\text { routing }=0\end{array}$ & 07 & 20 & 09 \\
\hline & & 20 & 37 & 17 \\
\hline
\end{tabular}

Calculated average value for each column will compare for each cell value in vertical manner and search for the minimum value (in case of transmission time, cost or incase of reliability maximum values will be consider) once this condition will match (i.e. average $<$ transmission time $\mid$ cost or average $>$ reliability), with the routing status is 0 for matched data cluster, the data cluster will gets distribute as per the selected route and routing status will set to 1 to ensure that data cluster will not be consider in the next iteration, otherwise logic will search for next appropriate match. By following the same routing mechanism first and third data clusters $\left(\mathrm{Cl}_{1}\left\{\mathrm{~s}_{11} * \mathrm{~s}_{13}\right\}\right.$ and $\left.\mathrm{Cl}_{2}\left\{\mathrm{~s}_{14}\right\}\right)$ will assign as mentioned in Table 10.

Table 10: Routed Clusters

\begin{tabular}{|l|l|l|}
\hline Processor & Clusters & Transmission Time \\
\hline $\mathrm{p}_{1}$ & $\mathrm{Cl}_{1}\left\{\mathrm{~s}_{11} * \mathrm{~s}_{13}\right\}$ & 18 \\
\hline $\mathrm{p}_{2}$ & $\mathrm{Cl}_{3}\left\{\mathrm{~s}_{14}\right\}$ & 20 \\
\hline
\end{tabular}

After completing routing process for data distribution of two data clusters, second data cluster $\left(\mathrm{Cl}_{2}\right)$ still remain unrouted as mentioned in Table 11. 
Table 11: showing unrouted cluster $\mathrm{Cl} 2$

\begin{tabular}{|c|c|c|c|c|}
\hline \multirow[t]{5}{*}{ CTTM () } & Clusters & $\mathrm{p}_{1}$ & $\mathrm{p}_{2}$ & $\mathrm{p}_{3}$ \\
\hline & $\underset{\text { routing=1 }}{\mathrm{Cl}_{1}\left\{\mathrm{~s}_{11} * \mathrm{~s}_{13}\right\}}$ & 18 & 45 & 24 \\
\hline & $\begin{array}{lll}\mathrm{Cl}_{2}\left\{\mathrm{~s}_{12}\right. & \left.* \mathrm{~s}_{15}\right\} \\
\text { routing }=0 & & \\
\end{array}$ & 35 & 48 & 18 \\
\hline & $\mathrm{Cl}_{3}\left\{\mathrm{~s}_{14}\right\}$ routing $=1$ & 07 & 20 & 09 \\
\hline & & 20 & 37 & 17 \\
\hline
\end{tabular}

Although $\mathrm{p}_{3}$ processor has minimum value in row 3 (09) with satisfying condition avg $<\mathrm{p}_{\text {time, }}$, But the data routing has already been done for these two rows as mentioned in Table 11. So finally data cluster $\left(\mathrm{Cl}_{2}\left\{\mathrm{~s}_{12} * \mathrm{~s}_{15}\right\}\right)$ will be route to $\mathrm{p}_{3}$ processor and overall transmission time is mentioned in Table 12.

Table 12: Overall Transmission Time

\begin{tabular}{|l|l|l|l|}
\hline Processor & Data signals & Transmission Time & ETime \\
\hline $\mathrm{p}_{1}$ & $\mathrm{~s}_{11} * \mathrm{~s}_{13}$ & 18 & \multirow{2}{*}{56} \\
\cline { 1 - 3 } $\mathrm{p}_{2}$ & $\mathrm{~s}_{14}$ & 20 & \\
\hline $\mathrm{p}_{3}$ & $\mathrm{~s}_{12} * \mathrm{~s}_{15}$ & 18 & \\
\hline
\end{tabular}

Overall transmission time for data packet $d_{1}$ is represent in graphical view as mentioned in Figure 3:

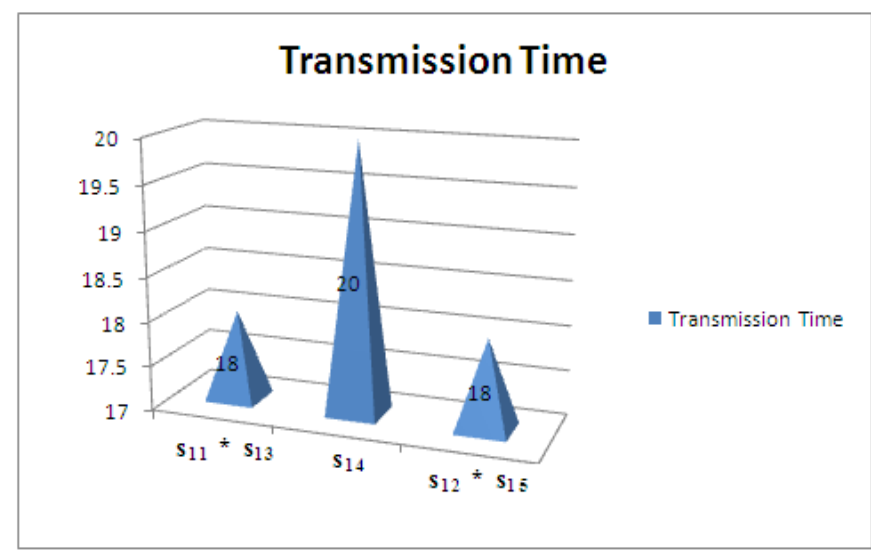

Fig 3: Overall transmission time

By applying the same routing mechanism for data distribution, this research paper would also calculate transmission cost and reliability for the given example in this research paper as mentioned in Table 13 and Table 14 respectively.

Table 13: Overall Transmission Cost

\begin{tabular}{|l|l|l|l|}
\hline Processor & Data Signals & Transmission cost & ECost \\
\hline $\mathrm{p}_{1}$ & $\mathrm{~s}_{21} * \mathrm{~s}_{24}$ & 190 & \multirow{2}{*}{905} \\
\cline { 1 - 3 } $\mathrm{p}_{2}$ & $\mathrm{~s}_{22} * \mathrm{~s}_{25}$ & 300 & \\
\hline $\mathrm{p}_{3}$ & $\mathrm{~s}_{23} * \mathrm{~s}_{26}$ & 415 & \\
\hline
\end{tabular}

Overall transmission cost for data packet $\mathrm{d} 2$ is represent in graphical view as mentioned in figure 4 :

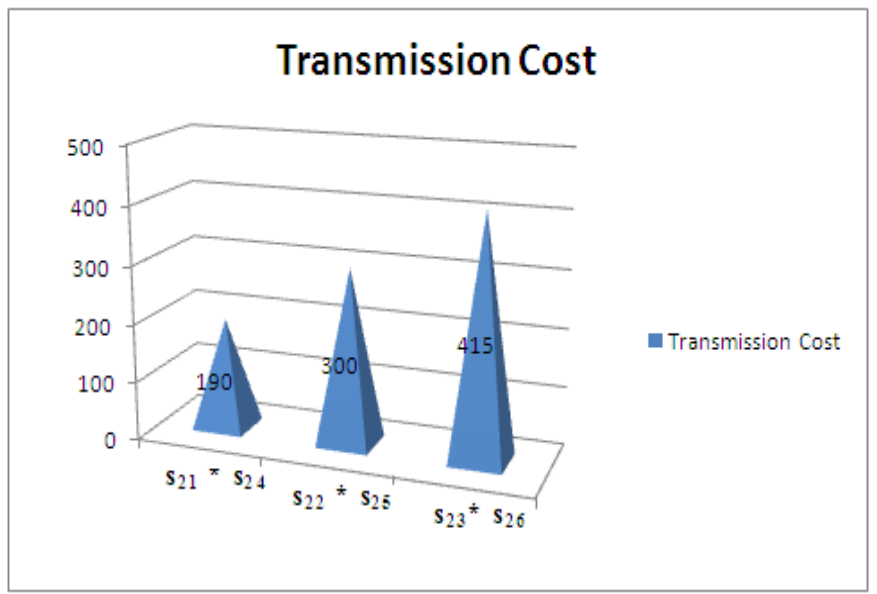

Fig 4: Overall Transmission Cost

Table 14: Overall Reliability

\begin{tabular}{|l|l|l|l|}
\hline Processor & Data Signals & Reliability & EReliability \\
\hline $\mathrm{p}_{1}$ & $\mathrm{~s}_{31} * \mathrm{~s}_{34} * \mathrm{~s}_{37}$ & 0.997965 & 0.975387 \\
\cline { 1 - 3 } $\mathrm{p}_{2}$ & $\mathrm{~s}_{32} * \mathrm{~s}_{33} * \mathrm{~s}_{36}$ & 0.978454 & \\
\cline { 1 - 3 } $\mathrm{p}_{3}$ & $\mathrm{~s}_{35} * \mathrm{~s}_{38}$ & 0.998899 & \\
\hline
\end{tabular}

Overall reliability for data packet $\mathrm{d}_{3}$ is represent in graphical view as mentioned in Figure 5:

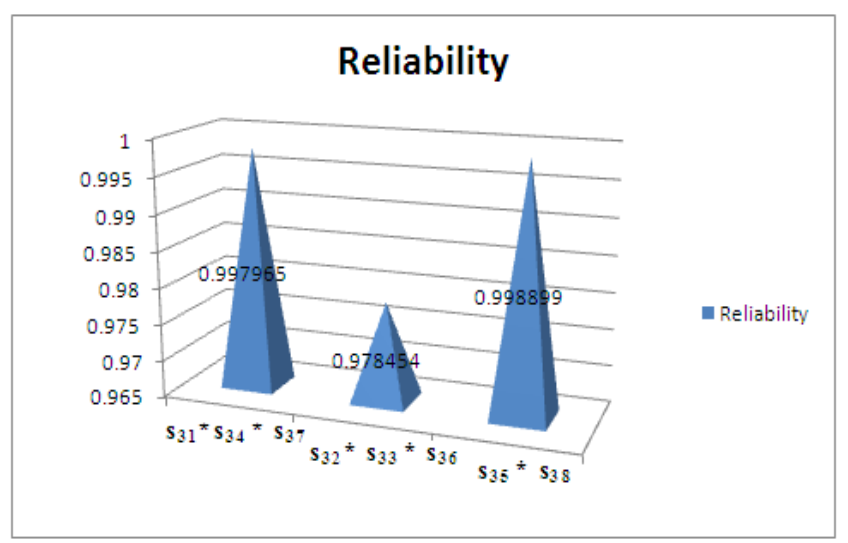

Fig 5: Overall Transmission Reliability

\section{CONCLUSION}

This research paper considers a data distribution problem in Mobile computing network. This problem solves by implementing a routing mechanism in order to achieve optimal data distribution through this research paper. Used routing mechanism will ensure to satisfy various constraints i.e. time, cost and reliability in MCN. This mechanism forms data clusters before routing, it makes the process simple and optimize. Data distribution routing mechanism is presented in pseudo code and applied on the several sets of input data to test the performance and effectiveness of the pseudo code. Optimization of data distribution is the common objective for any routing problem that the data packets needs to be route with optimal time, cost and reliability. This paper consider three data packets i.e. $d_{1}, d_{2}$ and $d_{3}$ with different data signals and transmission $d_{1}$ with minimum time, $d_{2}$ transmit with minimum cost and $d_{3}$ transmit with maximum reliability in $\mathrm{MCN}$. The optimal 
output of the given example that is consider in research paper to test the routing mechanism for data distribution is mentioned in the implementation section of the paper is mentioned in Table 15:

Table 15: Resultant Transmission Time, Cost and Reliability of given example

\begin{tabular}{|l|l|l|l|l|l|l|}
\hline Task & $\mathrm{p}_{1}$ & $\mathrm{p}_{2}$ & $\mathrm{p}_{3}$ & $\begin{array}{l}\text { Optimal } \\
\text { ETime }\end{array}$ & $\begin{array}{l}\text { Optimal } \\
\text { ECost }\end{array}$ & $\begin{array}{l}\text { Optimal } \\
\text { Ereliablity }\end{array}$ \\
\hline $\mathrm{d}_{1}$ & $\mathrm{~s}_{11} * \mathrm{~s}_{13}$ & $\mathrm{~s}_{14}$ & $\mathrm{~s}_{12} * \mathrm{~s}_{15}$ & 56 & --- & -- \\
\hline $\mathrm{d}_{2}$ & $\mathrm{~s}_{21} * \mathrm{~s}_{24}$ & $\mathrm{~s}_{22} * \mathrm{~s}_{25}$ & $\mathrm{~s}_{23} * \mathrm{~s}_{26}$ & --- & 905 & -- \\
\hline $\mathrm{d}_{3}$ & $\mathrm{~s}_{31} * \mathrm{~s}_{34} * \mathrm{~s}_{37}$ & $\mathrm{~s}_{32} * \mathrm{~s}_{33} * \mathrm{~s}_{36}$ & $\mathrm{~s}_{35} * \mathrm{~s}_{38}$ & --- & -- & 0.975387 \\
\hline
\end{tabular}

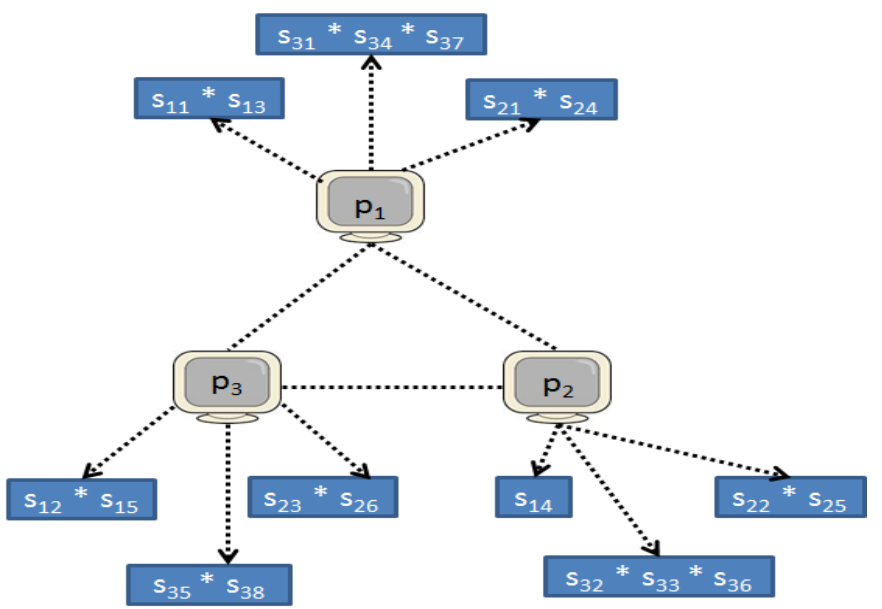

Fig 6: Data signals distribution at destination processing units in Mobile Computing

This research paper calculates time complexity of the present algorithm as it is a major factor to show the performance of the algorithm. Present paper also contains the comparison between results with some other recent algorithm [20] for proving the betterment of the present algorithm as mentioned in Table IX.

Table 9: Complexity comparison between present algorithm and algorithm [20]

\begin{tabular}{|l|l|l|l|}
\hline $\begin{array}{l}\text { Number } \\
\text { of } \\
\text { Processors } \\
(\mathrm{n})\end{array}$ & $\begin{array}{l}\text { Number } \\
\text { of data } \\
\text { Signals } \\
(\mathrm{m})\end{array}$ & $\begin{array}{l}\text { Complexity } \\
\text { of algorithm } \\
\left.[20] \text { O(n }{ }^{2}\right)\end{array}$ & $\begin{array}{l}\text { Complexity of } \\
\text { present } \\
\text { algorithm } \\
\text { O(mn })\end{array}$ \\
\hline 3 & 5 & 25 & 15 \\
\hline 3 & 6 & 36 & 18 \\
\hline 3 & 7 & 49 & 21 \\
\hline 3 & 8 & 64 & 24 \\
\hline 3 & 9 & 81 & 27 \\
\hline 4 & 5 & 25 & 20 \\
\hline 4 & 6 & 36 & 24 \\
\hline 4 & 7 & 49 & 28 \\
\hline 4 & 8 & 64 & 32 \\
\hline 4 & 9 & 81 & 36 \\
\hline 5 & 5 & 25 & 25 \\
\hline 5 & 6 & 36 & 30 \\
\hline 5 & 7 & 49 & 35 \\
\hline 5 & 8 & 64 & 40 \\
\hline 5 & 9 & 81 & 45 \\
\hline
\end{tabular}

Figure 7, 8 and 9 demonstrate the time complexity comparison between the present algorithm and algorithm [20] for different values $m$ and $n$.

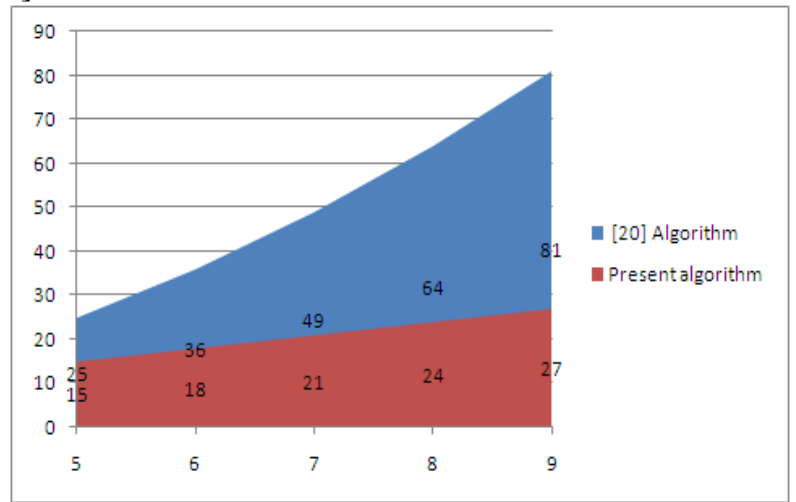

Fig 7: Number of Processors $=3$

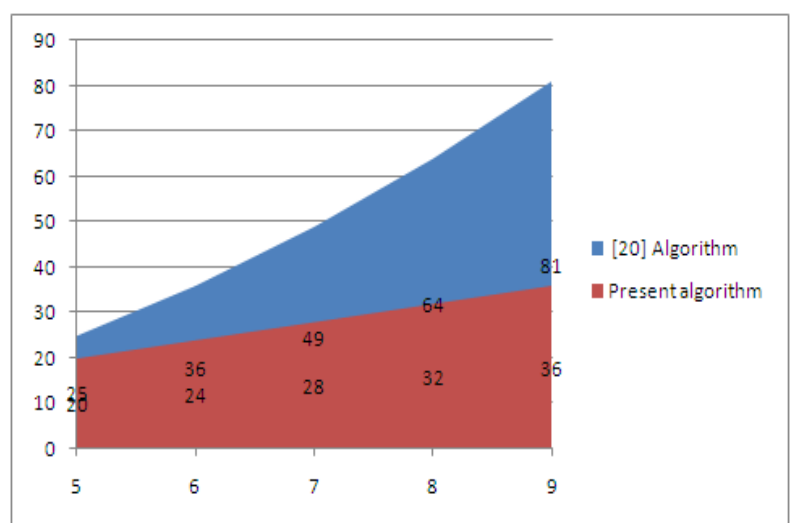

Fig 8: Number of Processors $=4$

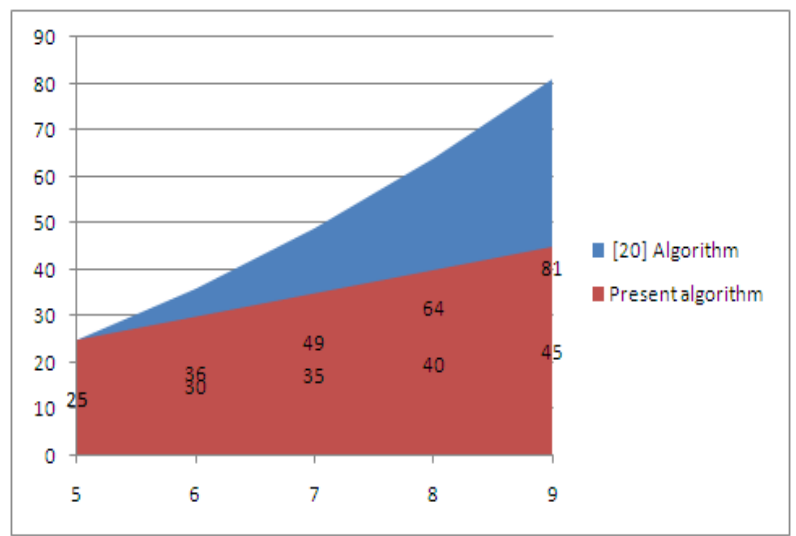

Fig 9: Number of Processors $=5$ 


\section{REFERENCES}

[1] Afzal Mawji, Hossam Hassanein, "Efficient content distribution for peer-to-peer overlays on mobile ad hoc networks", Journal of Advanced Research, Vol 2, Issue 3, pp. 265-279, 2011

[2] Agrakhed, J.; Biradar, G.S.; Mytri, V.D. "Adaptive Multi Constraint Multipath Routing Protocol in Wireless Multimedia Sensor Network", Computing Sciences (ICCS), 2012 International Conference on, On page(s): 326 - 331

[3] Bendjeddou, A.; Tolba, F.D.; Ghoualmi, N.; Lorenz, P. "DPCS: A distributed power control scheme for mobile wireless sensor networks", Innovation in Information \& Communication Technology (ISIICT), 2011 Fourth International Symposium on, On page(s): 30 - 34

[4] Biswas, K.; Muthukkumarasamy, V.; Sithirasenan, E.; Usman, M. "An energy efficient clique based clustering and routing mechanism in wireless sensor networks", Wireless Communications and Mobile Computing Conference (IWCMC), 2013 9th International, On page(s): 171 - 176

[5] Changjiang Jiang; Min Xiang; Weiren Shi "Overview of cluster-based routing protocols in wireless sensor networks", Electric Information and Control Engineering (ICEICE), 2011 International Conference on, On page(s): 3414 - 3417

[6] Deepika Kukreja, Umang and B V R Reddy. "Trust based Routing using Dominating Set Approach (TRDSA) in Wireless Ad-Hoc Networks", IJCA Proceedings on National Conference on Communication Technologies \& its impact on Next Generation Computing 2012 CTNGC(1), pp. 16-24, 2012

[7] E.M. Saad, M.H. Awadalla, and R.R. Darwish, "Energy aware distributed clustering scheme for wireless sensor networks, " International Journal of Computers, Issue 3, Vol. 2, 2008.

[8] Faizul Navi Khan, KapilGovil, "A TRICKY TASK SCHEDULING TECHNIQUE TO OPTIMIZE TIME COST AND RELIABILITY IN MOBILE COMPUTING ENVIRONMENT", International Journal of Research in Engineering and Technology, Vol 3, Issue 5, pp 1-7, 2014

[9] Faizul Navi Khan, KapilGovil, "An advanced Task Assignment Model to Optimize Time Cost and Reliability in Distributed Computing Environment", International Journal of Advanced Trends in Computer Science and Engineering, Vol 3, Issue 3, pp 23-28, 2014

[10] Faizul Navi Khan, Kapil Govil. "A Static approach to optimize time cost and reiliability in Distributed Processing Environment". International Journal of Scientific \& Engineering Research, Volume 05, Issue 5, Pages 1016-1021, 2014

[11] Faizul Navi Khan, Kapil Govil. "Cluster based optimization routing strategy for data communication in Mobile Computing". International Journal of Computer Applications, Volume 81, Issue 15, Pages 19-22, 2013
[12] Faizul Navi Khan, Kapil Govil, "Reliability Based Task Allocation Scheme to Enhance the Performance of Distributed Environment", International Journal of Computer Sciences and Engineering, Vol: 2 Issue: 8, pp: 99-102, 2014

[13] Faizul Navi Khan, Kapil Govil, "Static Approach for Efficient Task Allocation in Distributed Environment", International Journal of Computer Applications, vol: 81 issue: 15, pp: 19-22, 2013

[14] H. Zafar, D. Harle, I. Andonovic, Y. Khawaja, "Performance evaluation of shortest multipath source routing scheme", IET Commun, Vol. 3, Issue. 5, pp. 700-713, 2009

[15] J. N. AL-KARAKI and A. E. KAMAL, "Routing techniques in wireless sensor networks: a survey," IEEE Wireless Communications, Vol. 1, Issue 6, pp. 1536-1284, Dec 2004

[16] Jian Wang, Yanheng Liu, Yu Jiao, "Building a trusted route in a mobile ad hoc network considering communication reliability and path length", Journal of Network and Computer Applications, Vol. 34, Issue 4, pp. 1138-1149, 2011

[17] Karthik Subburathinam, "A Cluster Based Multipath Routing Protocol for Energy Conservation in Wireless Sensor Networks", European Journal of Scientific Research, Vol 78, No 4, pp. 559-569, 2012

[18] Kour, H. and A.K. Sharma, "Hybrid energy efficient distributed protocol for heterogeneous wireless sensor network", Int. J. Comput. Applications, Vol 4: pp 15. 2010

[19] Manisekaran, S.V., R. Venkatesan, "Distributed Cluster Based Routing Technique with Multiple Sinks for Wireless Sensor Networks", American Journal of Applied Science Vol 9, Issue 8, pp 12421250, 2012

[20] P. Ghosh, N. Roy, S. K. Das, "Mobility - aware efficient job scheduling in mobile grids", In: 7th IEEE Int. Symp. On Cluster Computing and the Grid, pp. 701-706, 14-17 May 2007

[21] Paolo Bellavista, Antonio Corradi, Mario Fanelli, Luca Foschini, "A survey of context data distribution for mobile ubiquitous systems", ACM Computing Surveys (CSUR), Volume 44 Issue 4, doi: 10.1145/2333112.2333119, 2012

[22] R.Chang and chia-juokuo, "An Energy Efficient Routing Mechanism for Wireless Sensor Networks," Proceedings of the 20th International Conference on Advanced Information Networking and Applications, Volume 02, pp. 308-12, 2006

[23] S.Kannan, S.Karthik, V.P. Arunachalam, "An Enhanced Packet Retransmission Method for Improving TCP-Aware Source Routing in Mobile Ad-Hoc Network", Asian Journal of Information Technology, Vol 10 Issue 1, pp. 20-25, 2011

[24] Tushar Agarwal, Raj Kumar, Pramod Sharma, SohanGarg, "Design \& Development of an Effective Routing Technique for Wireless Sensor Networks", International Journal of Advanced Research in Computer Science and Software Engineering, Vol 2, Issue 4, pp 386-389, 2012 